13,18

\title{
Магнитная анизотропия покрытых графеном ультратонких слоев железа на подложке из гексагонального нитрида бора $\mathrm{Gr} / \mathrm{Fe} / h$-BN
}

\author{
() С.М. Дунаевский ${ }^{1,2}$, E.K. Михайленко ${ }^{1,2}$ \\ ${ }^{1}$ Национальный исследовательский центр „Курчатовский институт“, \\ Петербургский институт ядерной физики им. Б.П. Константинова, \\ Гатчина, Россия \\ ${ }^{2}$ Санкт-Петербургский государственный электротехнический университет „ЛЭТИ“, \\ Санкт-Петербург, Россия \\ E-mail: smd2000@mail.ru
}

Поступила в Редакцию 10 июля 2020 г.

В окончательной редакции 10 июля 2020 г.

Принята к публикации 14 июля 2020 г

В рамках теории функционала спиновой плотности (SDFT) впервые представлены теоретические результаты компьютерного моделирования магнитных свойств ультратонких пленок железа на немагнитной подложке из гексагонального нитрида бора $\mathrm{Gr} / \mathrm{Fe} / h-\mathrm{BN}$. Основное внимание уделено численным исследованиям магнитной анизотропия пленок железа с гексагональной структурой, аналогичной кристаллической структуре интеркалированных пленок железа в системе $\mathrm{Gr} / \mathrm{Fe} / \mathrm{Ni}$ (111). Для этого рассчитаны полные энергии ферромагнитных структур с различными направлениями магнитных моментов атомов железа относительно поверхности пленки. Проанализировано влияние графена на магнитные свойства системы $\mathrm{Gr} / \mathrm{Fe} / h$-BN. Результаты теоретического моделирования $\mathrm{Fe} / h-\mathrm{BN}$ и $\mathrm{Gr} / \mathrm{Fe} / h$-BN показывают, что в такой системе можно получить достаточно „толстые“ пленки $\left(d_{\mathrm{Fe}} \geq 10 \AA\right)$ железа, которые будут обладать перпендикулярной магнитной анизотропией.

Ключевые слова: тонкие магнитные пленки, функционал спиновой плотности, магнитное диполь-дипольное взаимодействие, магнитная кристаллографическая анизотропия.

DOI: 10.21883/FTT.2020.11.50105.147

\section{1. Введение}

Одним из актуальных направлений в исследовании функциональных материалов является изучение ультратонких слоев ферромагнетиков (Fe и Co), обладающих перпендикулярной магнитной анизотропией („perpendicular magnetic anisotropy“, РМA) [1]. Такие монослои синтезируют путем интеркаляции магнитных атомов под графен, выращенный на поверхности (111) гексагонального никеля [2-5], оксиде кремния $\mathrm{SiO}[6,7]$, иридия $[8,9]$ и ряде других подложек. В $[10,11]$ нами были выполнены $a b$ initio расчеты магнитных свойств монослоев $\mathrm{Fe}$ и $\mathrm{Co}$, интеркалированных в систему $\mathrm{Gr} / \mathrm{Ni}(111)$ и обладающих fсc-структурой, свойственной никелю. Полученные результаты показали отсутствие PМА для пленок железа и кобальта на магнитной подложке из никеля, что обусловлено вкладом магнитного диполь-дипольного взаимодействия подложки. Перпендикулярная магнитная анизотропия в очень тонких пленках железа и кобальта (1-3 монослоя) возникала только тогда, когда подложка $\mathrm{Ni}$ (111) либо не учитывалась, либо задавалась немагнитной. В связи с этим, несомненный интерес представляет собой выполнение аналогичных расчетов энергии анизотропии ультратонких ферромагнитных пленок на подложке из немагнитного гексагонального нитрида бора $h$-BN, кристаллическая структура которого очень хорошо согласуется со структурой графена [12-14]. Так, расстояние между ближайшими атомами В и $\mathrm{N}$ в $h$-BN равно $1.44 \AA$, а расстояние между соседними атомами углерода в графене равно $1.42 \AA$. Несмотря на большое число работ, посвященных получению и исследованиям электронной структуры однои двуслойного графена на $h$-BN [12,15-17], работ по синтезу и изучению магнитных свойств пленок железа, интеркалированного в $\mathrm{Gr} / h$-BN довольно мало. Среди них следует отметить работу [18], где экспериментально рассмотрена интеркаляция одного монослоя железа в системе $\mathrm{Gr}-\mathrm{BN} / \mathrm{Fe} / \mathrm{Pt}(111)$. Полученные результаты свидетельствуют о возможности ситеза и более толстых пленок железа, покрытых нитридом бора, или графеном. В [19] выполнены $a b$ initio теоретические исследования туннельного магнетосопротивления суперструктуры $\mathrm{Fe} / \mathrm{BN} / \mathrm{Gr} / \mathrm{BN} / \mathrm{Pt}$, моделирующий туннельный переход на основе $h$-BN.

В настоящей работе в рамках SDFT $a b$ initio впервые исследуется магнитная анизотропия покрытых графеном пленок железа на подложке из гексагонального нитрида бора - $\mathrm{Gr} / \mathrm{Fe}(\mathrm{N}) / h$-BN. Здесь $N$ - число монослоев железа, образующих гексагональную плотно упакованную структуру.

\section{2. Метод расчета}

Ab initio расчеты полной энергии системы для различных направлений оси квантования спинового момента 
были выполнены в рамках метода псевдопотенциала, реализованного в пакете Quantum Espresso [20]. Суперъячейка включала в себя шесть слоев гексагонального нитрида бора, от одного до шести слоев железа и два атома углерода (20 атомов для $\mathrm{Gr} / \mathrm{Fe}(6) / h$-BN). В расчетах предполагалось, что монослои железа обладают той же гексагональной плотно упакованной структурой, что и в системе $\mathrm{Gr} / \mathrm{Fe} / \mathrm{Ni}$ (111) [10,11]. Расстояние между верхним слоем атомов железа и графеном было принято равным $2.04 \AA$. Ширина вакуумного зазора равнялась 14 А̊. Подложка всегда моделировалась шестью монослоями $h$-BN, расстояние между которыми равнялось $3.25 \AA$ [17]. Начальные конфигурации валентных электронов имели вид: $2 s 2 p(\mathrm{~B}), 2 s 2 p^{2}(\mathrm{C}), 2 s 2 p^{3}(\mathrm{~N})$, $3 s^{2} p^{6} 3 d^{6} 4 s^{2}(\mathrm{Fe}), 3 s^{2} p^{6} 3 d^{7} 4 s^{2}(\mathrm{Co})$.

На первом этапе были определены полные энергии супеъячейки для различных вариантов расположения монослоев железа на $h$-BN. Оказалось, что минимальной энергии отвечают конфигурации с расположением атомов железа непосредственно над атомами азота. Для одного монослоя Fе кристаллическая структура системы $\mathrm{Fe} / h$-BN показана на рис. 1. Для двух монослоев железа существует уже два варианта относительного расположения монослоев с примерно одинаковой полной энергией. Энергии суперъячеек всех остальных конфигураций атомов железа на нитриде бора оказываются выше примерно на $0.8-1 \mathrm{eV}$.

Кроме того, существует еще несколько вариантов расположения графена на монослоях железа. Для системы

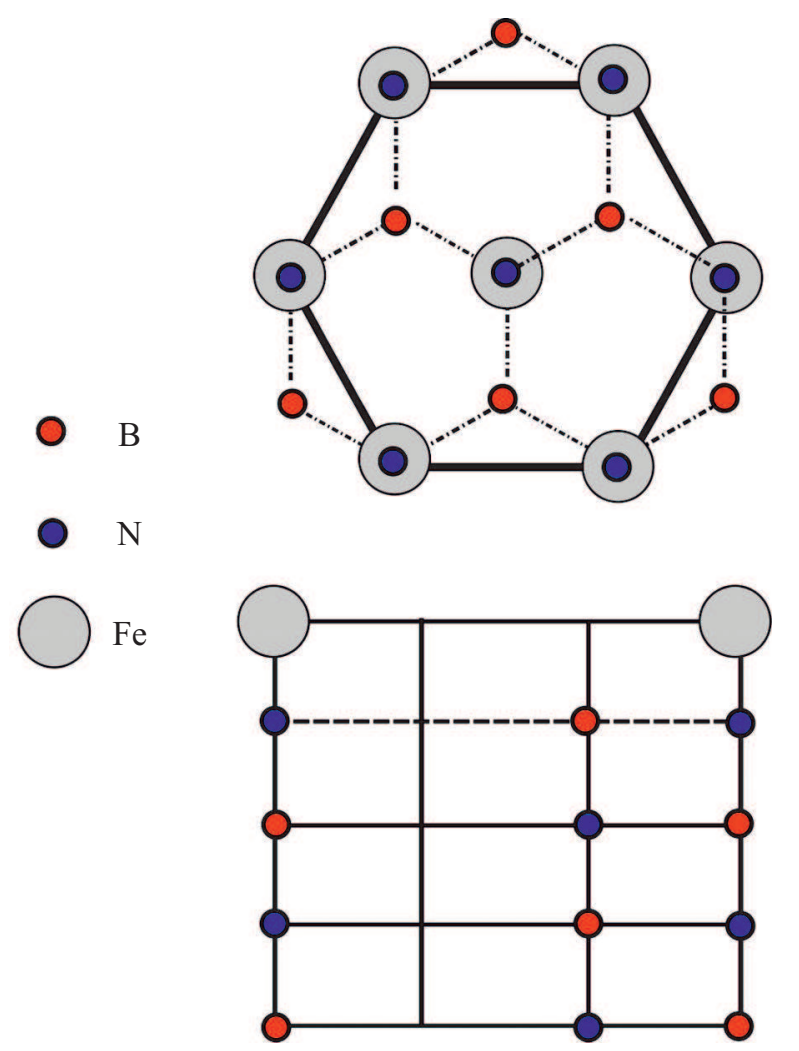

Рис. 1. Кристаллическая структура системы $\mathrm{Fe} / h$-BN, обладающая минимальной полной энергией.
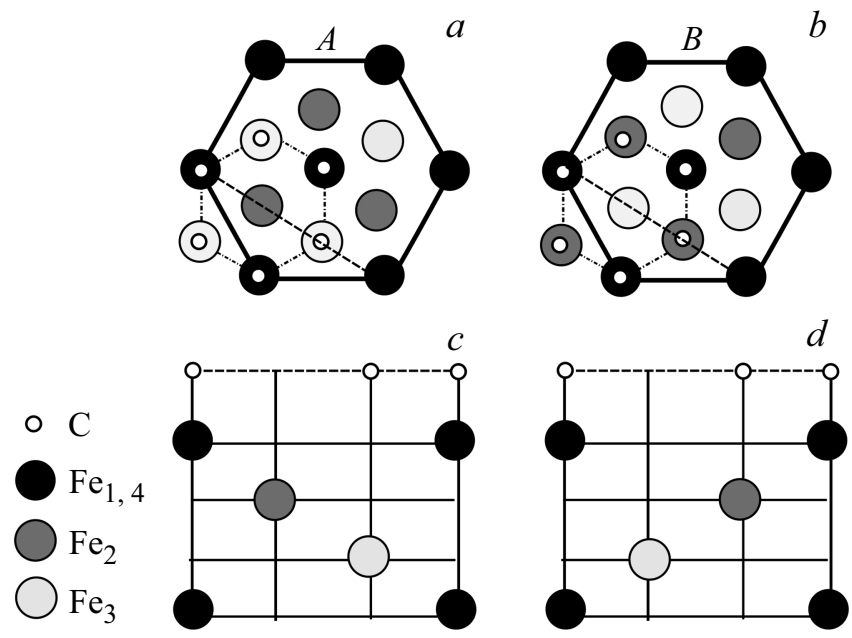

Рис. 2. Варианты $A$ и $B$ кристаллической структуры системы $\mathrm{Gr} / \mathrm{Fe}(4) / h$-BN: $a, b-$ вид сверху; $c, d-$ вид в сечении, показанном на рис. $a$ и $b$ пунктиром.

с четырьмя монослоями железа $\mathrm{Gr} / \mathrm{Fe}(4) / h$-BN на рис. 2 изображены две структуры $A$ и $B$ с практически одинаковыми полными энергиями. Нумерация монослоев железа начинается с верхнего монослоя, граничащего с графеном. Атом железа с максимальным значением $N$ (на границе с подложкой) всегда расположен непосредственно над атомом азота. В этих структурах три атома углерода всегда расположены над атомами железа ближайшего монослоя, а еще три атома С находятся над атомами Fе более глубоких монослоев.

Такие же конфигурации возникают и в системе $\mathrm{Fe}(\mathrm{N}) / h-\mathrm{BN}$, когда число монослоев железа $N \geq 2$. Численные расчеты показали, что в системе $\mathrm{Gr} / \mathrm{Fe}(\mathrm{N}) / h$-BN для значений $N=1,2$ минимальной полной энергией обладает $A-$ структура, а для $N \geq 3-B$-структура. Энергии элементарных ячеек $A$ - и $B$-структур при этом отличаются друг от друга не более чем $10 \mathrm{meV}$. Энергии остальных допустимых положений графена на г.п.у.-решетке железа оказываются примерно на $0.5 \mathrm{eV}$ больше энергий $A$ - и $B$-структур. Поэтому, результаты расчетов энергии анизотропии этих структур в статье не приводятся.

Для самосогласованного вычисления спектра $\varepsilon_{v, \sigma}(k)$ использовалось обобщенное градиентное разложение GGA [21] обменно-корреляционного функционала. Разложение волновых функций валентных электронов на плоские волны ограничивалось энергией $300 \mathrm{Ry}$. В качестве псевдопотенциалов были выбраны полностью релятивистские PAW псевдопотенциалы Perdew-Zunger [22]. При суммировании по зоне Бриллюэна использовалось разбиение Monkhorst-Pack $12 \times 12 \times 1$. Полная энергия суперячейки вычислялась с точностью $10^{-6} \mathrm{eV}$.

В методе SDFT вычислялась полная энергия рассматриваемых систем

$$
E^{\text {tot }}=E^{\text {band }}-E^{d c}+E^{m d}
$$


где

$$
\begin{gathered}
E^{\text {band }}=\sum_{\sigma, v, k} \varepsilon_{v, \sigma}(k)=\int^{\varepsilon_{F}} \varepsilon n_{\sigma}(\varepsilon) d \varepsilon, \\
E^{m d}=\frac{1}{2} \sum_{i, k} \frac{\mu_{i} \mu_{k} R_{i k}^{2}-3\left(\mu_{i} R_{i k}\right)\left(\mu_{k} R_{i k}\right)}{R_{i k}^{5}} .
\end{gathered}
$$

Здесь $E^{\text {band }}$ - одночастичная зонная энергия, $E^{d c}-$ многочастичная „,double counting“ энергия [23], которая появляется в ходе самосогласованных расчетов уравнений Кона-Шэма, $E^{m d}-$ классическая энергия магнитного диполь-дипольного взаимодействия.

При учете спин-орбитального взаимодействия („spinorbital interaction“, SOI) полная энергия $E^{t o t}(\theta, \varphi)$ зависит от двух углов $\theta$ и $\varphi$, которые задают направление оси квантования спина. В работе рассматривались только коллинеарные ферромагнитные структуры, для которых зависимость зонной энергии от азимутального угла $\varphi$ не учитывалась. Заметим, что все рассмотренные структуры обладают осью симметрии третьего порядка $\mathrm{C}_{3}$, перпендикулярной поверхности. Для коллинеарных магнитных структур с заданной осью квантования, самосогласованным образом вычислялись значения спектра $\varepsilon_{v, \sigma}(k)$ и одночастичные состояния $\varphi_{v, \sigma}(k)$. Из этих данных находились плотности состояний $(\mathrm{DOS}) n_{\sigma}(\varepsilon)$, числа заполнения всех атомных орбиталей в ячейке и атомные магнитные моменты $\mu_{i}$. При выполнении численных вычислений $E^{m} d(\theta)$ учитывалось не менее 100 координационных сфер, что обеспечило очень хорошую сходимость результатов расчета и требуемую точность определения $E^{m d}\left(10^{-6} \mathrm{eV}\right)$. Для определения энергии системы при изменении направления намагниченности (оси квантования) использовалась теорема [21] (,local force theorem“), согласно которой изменением $E^{d c}$ в первом порядке по углу $\theta$ можно пренебречь. Поэтому, в работе вычислялись только зависимости $E^{\text {band }}(\theta)$ и $E^{m d}(\theta)$, где $\theta-$ угол между осью $Z$, перпендикулярной подложке, и направлением магнитного момента $\mu_{i}$.

Зависимость зонной энергии от полярного угла $E^{\text {band }}(\theta)$ может быть хорошо описана выражением, содержащим две константы анизотропии

$$
E^{\text {band }}(\theta)=K_{0}^{\mathrm{Fe}}+K_{1}^{\mathrm{Fe}} \sin ^{2} \theta+K_{2}^{\mathrm{Fe}} \sin ^{4} \theta .
$$

Здесь постоянная $r_{0}^{\mathrm{Fe}}$ - максимальное значение полной энергии системы, которая всегда отрицательна. Кроме того, $\left|K_{0}\right| \gg K_{1}, K_{2}$. Ввиду большой трудоемкости вычислений всей зависимости $E^{\text {band }}(\theta)$ при изучении магнитной анизотропии обычно (см. [1]) рассматривают разности энергий

$$
\Delta E^{\text {band }}=E_{\perp}^{\text {band }}-E_{\|}^{\text {band }} \text { и } \Delta E^{m d}=E_{\perp}^{m d}-E_{\|}^{m d},
$$

где индексы $\perp$ и $\|$ соответствуют углам $\theta=0^{\circ}$ и $\theta=90^{\circ}$. Отрицательные значения величин $\Delta E^{\text {tot }}=\Delta E^{\text {band }}+\Delta E^{m d}$ свидетельствуют о возникновении в системе перпендикулярной поверхности одноосной анизотропии. Заметим, что вклад диполь-дипольного взаимодействия $E^{m d}$ в полную энергию для плоских ультратонких слоев всегда отрицателен и максимален в том случае, когда все диполи лежат в плоскости слоя. Поэтому, для всех рассмотренных систем $\Delta E^{m d}>0$.

\section{3. Результаты расчетов и их обсуждение}

На первом этапе исследования были вычислены магнитные моменты атомов железа $\mu_{i}^{\mathrm{Fe}}$, которые необходимы для определения $E^{m d}(\theta)$ различных кристаллических структур. Здесь индекс $i$ нумерует монослои железа, начиная с верхнего. Для двух вариантов расположения атомов железа на нитриде бора в системе $\mathrm{Fe}(\mathrm{N}) / h-\mathrm{BN}$ значения $\mu_{i}^{\mathrm{Fe}}$ (в магнетонах Бора) представлены в табл. 1. Подложка из нитрида бора всегда моделировалась шестью слоями гексагонального BN. Из табл. 1 следует, что значения магнитных моментов двух конфигураций $A$ - и $B$-системы $\mathrm{Gr} / \mathrm{Fe}(6) / h$-BN для всех слоев незначительно отличаются друг от друга. В качестве примера влияния графена на атомные магнитные моменты Fе в табл. 2 приведены значения этих моментов для энергетически наиболее выгодной конфигурации $B$ в $\mathrm{Gr} / \mathrm{Fe}(6) / h-\mathrm{BN}$. Еще раз подчеркнем, что магнитные моменты железа рассчитаны для ферромагнитной гексагональной плотно упакованной структуры, которая ранее была экспериментально обнаружена на поверхности $\mathrm{Ni}(111)$ [2-5]. Аналогичные результаты получены для конфигурации $B$ и еще порядка шести возможных вариантов структуры $\mathrm{Gr} / \mathrm{Fe}(\mathrm{N}) / h$-BN.

Типичные зависимости энергии $E^{b a n d}$ от угла $\theta$ между нормалью к поверхности пленки железа и направлением ее спонтанного намагничивания аналитически хорошо

Таблица 1. Величины магнитных моментов атомов железа $\mu_{i}^{\mathrm{Fe}}$ $\left(\mu_{\mathrm{B}}\right)$ конфигураций $A$ и $B$ в системе $\mathrm{Fe}(\mathrm{N}) / h$-BN.

\begin{tabular}{c|c|c|c|c|c|c}
\hline$\mu_{i}^{\mathrm{Fe}}-B$ & $\mu_{1}$ & $\mu_{2}$ & $\mu_{3}$ & $\mu_{4}$ & $\mu_{5}$ & $\mu_{6}$ \\
\hline$N=1$ & 2.28 & - & - & - & - & - \\
$N=2$ & 1.58 & 1.08 & - & - & - & - \\
$N=3$ & 1.28 & 1.09 & 1.01 & - & - & - \\
\hline$\mu_{i}^{\mathrm{Fe}}-A$ & $\mu_{1}$ & $\mu_{2}$ & $\mu_{3}$ & $\mu_{4}$ & $\mu_{5}$ & $\mu_{6}$ \\
\hline$N=1$ & 2.28 & - & - & - & - & - \\
$N=2$ & 1.77 & 1.25 & - & - & - & - \\
$N=3$ & 1.71 & 0.45 & 1.16 & - & - & -
\end{tabular}

Таблица 2. Величины магнитных моментов атомов железа

\begin{tabular}{c|c|c|c|c|c|c}
\hline$\mu_{i}^{\mathrm{Fe}}-B$ & $\mu_{1}$ & $\mu_{2}$ & $\mu_{3}$ & $\mu_{4}$ & $\mu_{5}$ & $\mu_{6}$ \\
\hline$N=1$ & 0.91 & - & - & - & - & - \\
$N=2$ & 0.46 & 0.60 & - & - & - & - \\
$N=3$ & 1.22 & 0.88 & 1.23 & - & - & -
\end{tabular}



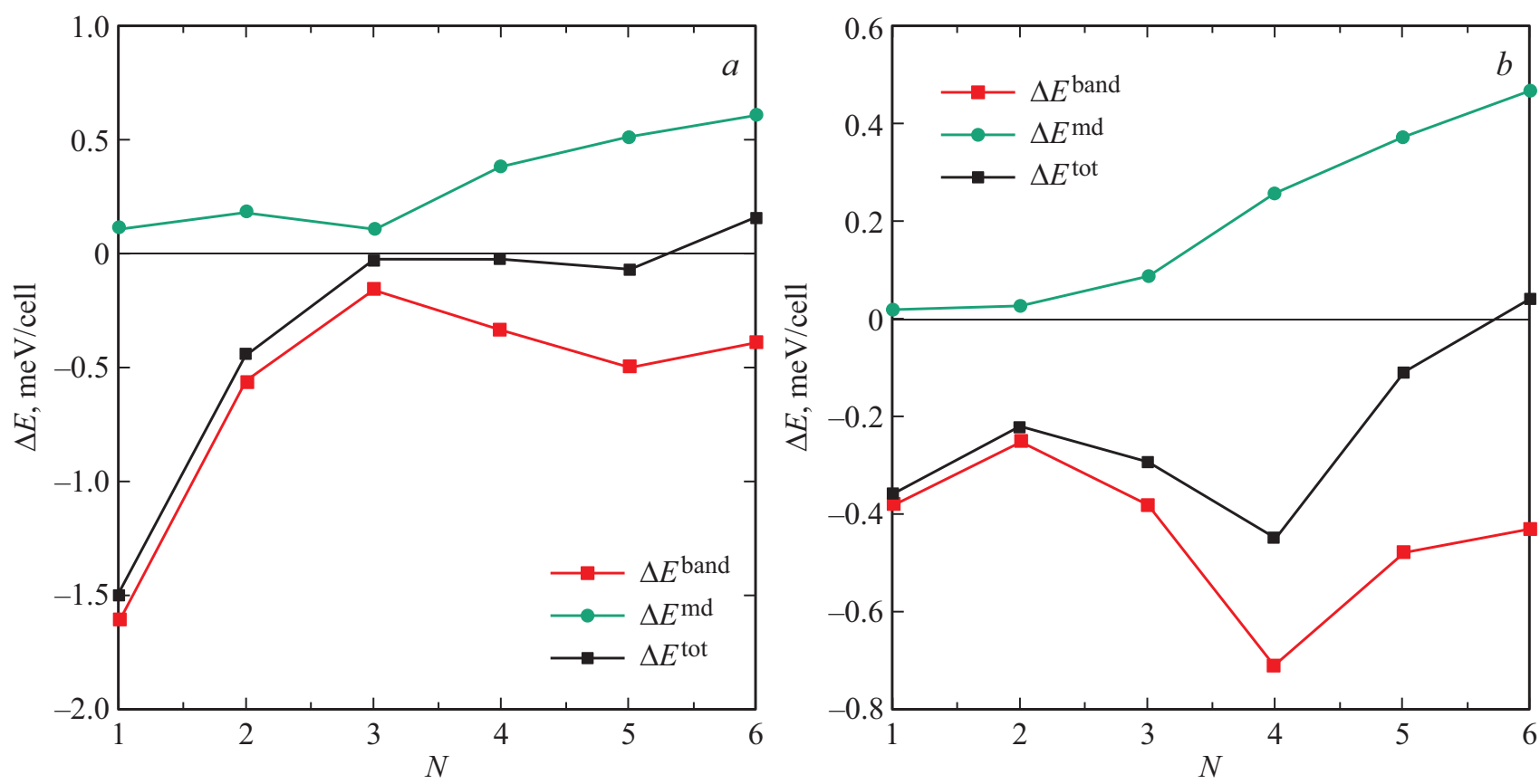

Рис. 3. Зависимость различных вкладов в полную энергию конфигурации $B$ от числа монослоев железа $N$ : $a) \mathrm{Fe}(\mathrm{N}) / h$ - $\mathrm{BN}$, b) $\mathrm{Gr} / \mathrm{Fe}(\mathrm{N}) / h$-BN. Результаты вычислений (точки) соединены линиями.

описываются выражением (4) с $K_{1}^{\mathrm{Fe}}, K_{2}^{\mathrm{Fe}}>0$. Таким образом, $\Delta E^{\text {band }}$ нашей системы всегда отрицательно. Для $\mathrm{Fe} / \mathrm{BN}$ достаточно одной константы $K_{1}^{\mathrm{Fe}}=0.37 \mathrm{meV} / \mathrm{cell}$ $\left(\sim 0.7 \mathrm{erg} / \mathrm{cm}^{2}\right)$, т. к. $K_{2}^{\mathrm{Fe}} \ll K_{1}^{\mathrm{Fe}}$. Это значение $K_{1}^{\mathrm{Fe}}$ монослоя железа на подложке из нитрида бора оказалось меньше значения аналогичной константы анизотропии свободного монослоя железа, вычисленного в [10]. Для $\mathrm{Gr} / \mathrm{Fe}(\mathrm{N}) / \mathrm{BN}(N>1)$ необходимо вводить две константы анизотропии. Так, для $\mathrm{Gr} / \mathrm{Fe}(2) / \mathrm{BN}$ подгонка дает значения $K_{1}^{\mathrm{Fe}} \sim K_{2}^{\mathrm{Fe}}=0.72 \mathrm{meV} /$ cell $\left(\sim 1.4 \mathrm{erg} / \mathrm{cm}^{2}\right)$. Значения магнитных моментов всех атомов железа $\mu_{i}^{\mathrm{Fe}}$ были использованы для нахождения энергии диполь-дипольного взаимодействия в рассматриваемых системах при значениях углов $\theta=0^{\circ}$ и $\theta=90^{\circ}$. Численные вычисления $E^{m d}(\theta)$ учитывали не менее 100 координационных сфер, что обеспечило очень хорошую сходимость результатов расчета и точность определения $E^{m d}$ не менее $10^{-6} \mathrm{eV}$. Контакт графена с поверхностью пленки меняет магнитные моменты атомов, находящихся в четырех верхних слоях. При этом в первом слое они понижаются, а во втором и третьем, наоборот, повышаются. Такое перераспределение магнитных моментов в пленке железа практически не меняет величину $\Delta E^{m d}$ для системы $\mathrm{Gr} / \mathrm{Fe}(6) / \mathrm{BN}$ в отличии от свободных пленок железа, покрытых графеном $\mathrm{Gr} / \mathrm{Fe}[10]$. Изменение величин $\Delta E^{\text {band }}$, $\Delta E^{m d}$ и $\Delta E^{t o t}$ в зависимости от числа $N$ монослоев $\mathrm{Fe}$, показано на рис. 3.

Из этого рисунка следует, что PMA $\left(\Delta E^{t o t}<0\right)$ в пленках железа сохраняется вплоть до пяти монослоев $(N=5)$. Графен несколько увеличивает константу анизотропии для $N \leq 5$, но не изменяет критическое значение $N=6$ при котором магнитно-дипольное взаимодействие меняет знак изменения полной энергии. Таким образом, существует критическая толщина пленки железа с перпендикулярной магнитной анизотропией PMA. В более толстых пленках ориентация магнитного момента системы становится продольной (в плоскости пленки, $\Delta E^{t o t}>0$ ), а PMA изменяется на (,in plane“ магнитную анизотропию IMA. Аналогичные результаты для A-структуры практически не отличаются от результатов, представленных на рис. 3 .

\section{4. Заключение}

В настоящей работе методом SDFT впервые представлены теоретические результаты компьютерного моделирования магнитных свойств ультратонких пленок железа на подложке из гексагонального нитрида бора. Наибольшее внимание уделено численным исследованиям магнитной анизотропия пленок железа и выяснению роли графена в модельной системе $\mathrm{Gr} / \mathrm{Fe}(\mathrm{N}) / h$ - $\mathrm{BN}$. Для этого были определены полные энергии системы для двух направлений оси квантования (перпендикулярно и вдоль поверхности пленки) и рассчитаны магнитные моменты всех атомов. Результаты теоретического моделирования $\mathrm{Fe}(\mathrm{N}) / h-\mathrm{BN}$ и $\mathrm{Gr} / \mathrm{Fe}(\mathrm{N}) / h$ - $\mathrm{BN}$ позволяют высказать предположение, что в такой системе можно получить достаточно „толстые“ пленки $\left(d_{\mathrm{Fe}} \geq 10 \AA, N \approx 6\right)$ железа, которые будут обладать РМА. При дальнейшем увеличении числа монослоев железа $(N>6)$ в рассмотренных системах происходит переход от РМА к IMA. Показано, что хотя графен и изменяет атомные моменты 
системы, это изменение не влияет на критическую толщину пленки железа, при которой изменяется характер магнитной анизотропии. Поскольку графен защищает железо от воздействия атмосферного кислорода, такие пленки могут оказаться перспективными для практических приложений.

\section{Благодарности}

Авторы выражают благодарность „Центру обработки данных РК ПИК“ НИЦ „Курчатовский институт, ПИЯФ за предоставленные вычислительные ресурсы.

\section{Конфликт интересов}

Авторы заявляют, что у них нет конфликта интересов.

\section{Список литературы}

[1] B. Dieny, M. Chshiev. Rev. Mod. Phys. 89, 025008 (2017).

[2] G. Bertoni, L. Calmels, A. Altibelli, V. Serin. Phys. Rev. B 71, 075402 (2004).

[3] Y.S. Dedkov, M. Fonin, U. Rudiger, C. Laubschat. Appl. Phys. Lett. 93, 022509 (2006).

[4] Yu.S. Dedkov, M. Fonin. New J. Phys. 12, 125004 (2010).

[5] G.S. Grebenyuk, S.M. Dunaevsky, E.Yu. Lobanova, D.A. Smirnov, I.I. Pronin. Appl. Surf. Sci. 470, 840 (2019).

[6] C. Berger, Z. Song, T. Li, X. Li, A.Y. Ogbazghi, R. Feng, Z. Dai, A.N. Marchenkov, E.H. Conrad, O.N. First, W.A. de Heer. J. Phys. Chem. B 108, 19912 (2004).

[7] C. Riedl, C. Coletti, T. Iwasaki, A.A. Zakharov, U. Starke. Phys. Rev. Lett. 103, 246804 (2009).

[8] N. Rougemaille, A.T. N'Diaye, J. Coraux, C. Vo-Van, O. Fruchart, A.K. Schmid. Appl. Phys. Lett. 101, 142403 (2012).

[9] A.D. Vu, J. Coraux, G. Chen, A.T. N'Diaye, A.K. Schmid, N. Rougemaille. Sci. Rep. 6, 24783 (2015).

[10] S. M. Dunaevskii, E.Yu. Lobanova, E.K. Mikhailenko, I.I. Pronin. Phys. Solid State 61, 1310 (2019).

[11] S.M. Dunaevsky, E.K. Mikhailenko, I.I. Pronin. Adv. Mater. Lett. 10, 633 (2019).

[12] C. Oshima, A. Itoh, E. Rokuta, T. Tanaka, K. Yamashita, T. Sakurai. Solid State Commun. 116, 37 (2000).

[13] T. Kawasaki, T. Ichimura, H. Kishimoto, A.A. Akbar, T. Ogawa, C. Oshima. Surf. Rev. Lett. 09, 1459 (2002).

[14] T. Tanaka, A. Itoh, K. Yamashita, E. Rokuta, C. Oshima. Surf. Rev. Lett. 10, 697 (2003).

[15] Y. Sakai, S. Saito, M.L. Cohen. J. Phys. Soc. Jpn. 84, 121002 (2015).

[16] Y. Cao, V. Fatemi, S. Fang, K. Watanabe, T. Taniguchi, E. Kaxiras, P. Jarillo-Herrero. Nature 556, 44 (2018).

[17] G. Giovannetti, P.A. Khomyakov, G. Brocks. P.J. Kelly, J. van den Brink. Phys. Rev. B 76, 073103 (2007).

[18] I. Pǐ̌, S. Nappini, F. Bondino, T.O. Menteş, A. Sala, A. Locatelli, E. Magnano. Carbon 134, 274 (2018).

[19] A.M. Ukpong. J. Phys. D 51, 095302 (2018).

[20] P. Giannozzi, O. Andreussi, T. Brumme, O. Bunau, M.B. Nardelli, M. Calandra, R. Car, C. Cavazzoni, D. Ceresoli, M. Cococcioni, N. Colonna. J. Phys. Condens. Matter. 29, 395502 (2009).
[21] J.P. Perdew, J.A. Chevary, S.H. Vosko, K.A. Jackson, M.R. Pederson, D.J. Singh, C. Fiolhais. Phys. Rev. B 46, 6671 (1992).

[21] J.P. Perdew, A. Zunger. Phys. Rev. B 23, 5048 (1981).

[22] A.I. Liechtenstein, M.I. Katsnelson, V.P. Antropov, V.A. Gubanov. J. Magn. Magn. Mater. 67, 65 (1987).

Редактор Т.Н. Василевская 